\title{
Effect of Taurine on Biliary Excretion and Metabolism of Acetaminophen in Male Hamsters
}

\author{
Ja Yong Lee, ${ }^{a}$ Da Woon Jung, ${ }^{a}$ Hyun Ah PArk, ${ }^{a}$ Soo Jeong KIm, ${ }^{a}$ Jin Ho Chung, ${ }^{a}$ \\ Chang Kiu Moon, ${ }^{a}$ and Young Chul KIM*,a,b \\ ${ }^{a}$ College of Pharmacy, Seoul National University; and ${ }^{b}$ Research Institute of Pharmaceutical Sciences, Seoul National \\ University; San 56-1 Shinrim-Dong, Kwanak-Ku, Seoul 151-742, Republic of Korea. \\ Received June 14, 2004; accepted August 6, 2004
}

The effect of taurine intake on the biliary disposition and toxicity of acetaminophen (APAP) was examined in male Golden-Syrian hamsters. Animals were provided with taurine (5 mM) in drinking water for 1 week followed by APAP treatment $(250 \mathrm{mg} / \mathrm{kg}$, i.p.). Biliary excretion and plasma concentrations of APAP and its major metabolites were determined for up to $360 \mathrm{~min}$. Taurine increased the bile flow, whereas the concentration of APAP or the metabolites in bile was not altered significantly. Accordingly the total biliary excretion of APAP and the metabolites was increased in hamsters fed taurine. Taurine increased the plasma concentrations of APAPglutathione (GSH) and APAP-mercapturate, but the APAP-glucuronide or APAP-sulfate concentration was not changed. The area under the curve of the plasma APAP concentration was reduced significantly, suggesting that the elimination of APAP was enhanced by taurine intake. However, the hepatotoxicity resulting from a dose of APAP $(450 \mathrm{mg} / \mathrm{kg}$, i.p.) was not altered by taurine intake as determined by the elevation of serum alanine aminotransferase, aspartate aminotransferase, and sorbitol dehydrogenase activities. The results suggest that taurine administration could affect the disposition of APAP by enhancing its metabolism through the GSH-dependent pathway and also by increasing the biliary excretion of this drug and its metabolites. The pharmacological significance of this finding remains to be examined.

Key words taurine; acetaminophen; bile flow; biliary excretion; hepatotoxicity

Taurine or 2-aminoethanesulfonic acid is a $\beta$-amino acid that is one of the most abundant free amino acids in many mammalian tissues. Its physiological functions have been proposed to include antioxidation, detoxicification, osmoregulation, membrane stabilization, and neuromodulation. Also taurine is conjugated with bile acids in liver to form bile salts that promote lipid absorption from small intestine.

It was reported that taurine intake increased the bile flow and bile acid output in hamsters and guinea pigs. ${ }^{1,2)}$ Since taurine was shown to increase the hepatic cholesterol $7 \alpha$ hydroxylase and 3-hydroxy-3-methylglutaryl coenzyme A (HMG CoA) reductase activities, the increase in bile flow has been accounted for, at least in part, by the increment in bile acid synthesis. It has also been suggested that the activities of these two enzymes would be regulated by a sulfhydryl substance such as cysteine and glutathione (GSH) which is closely related to the biosynthesis of taurine. ${ }^{3,4)}$

Acetaminophen (APAP), a widely used analgesic-antipyretic, is detoxified through the formation of sulfate and glucuronide conjugates. At higher doses, however, APAP is increasingly converted to a reactive metabolite, $N$-acetyl- $p$ benzoquinoneimine (NAPQI), by cytochrome P450 (CYP)mediated reactions in which CYP1A2, CYP2E1, and CYP3A4 play critical roles., ${ }^{5,6}$ The reactive electrophilic metabolite is detoxified by conjugation with hepatic GSH, and the resulting APAP-GSH conjugate is mostly excreted through bile. ${ }^{7)}$ When the generation of electrophilic metabolites exceeds the availability of hepatic GSH for conjugation, covalent binding of the metabolites to cellular macromolecules may result, an event that correlates with hepatic necrosis. Therefore, the hepatotoxicity of APAP is dependent on the metabolic activities responsible for activation of this drug and the effectiveness of GSH conjugation reactions.

In this study we determined the elimination of APAP in hamsters fed taurine in drinking water for 1 week. Since biliary excretion and GSH-dependent metabolic reactions play critical roles in the disposition of APAP, and both could be influenced by taurine, it was of interest to examine the effects of taurine intake on the disposition and toxicity of this analgesic-antipyretic.

\section{MATERIALS AND METHODS}

Animals and Treatments Male Golden-Syrian hamsters (120-150 g) were purchased from Japan SLC (Tokyo, Japan). The use of hamsters was in compliance with the guidelines established by the institutional animal care committee. Animals were housed in temperature $\left(22 \pm 2^{\circ} \mathrm{C}\right)$ and humidity $(55 \pm 5 \%)$ controlled rooms with a $12 \mathrm{~h}$ light : dark cycle (light: 0800-2000). Regular lab chow and tap water were allowed ad libitum. Taurine-dissolved tap water $(5 \mathrm{~mm}$; $0.063 \mathrm{~g} / 100 \mathrm{ml}$ ) replaced normal tap water for 1 week prior to the experiment. There was no difference in intake of water, feed, or body weight gain between control and taurine-fed hamsters during the period of taurine feeding.

Hamsters were anesthetized with an intraperitoneal dose $(80 \mathrm{mg} / \mathrm{kg})$ and an oral dose $(80 \mathrm{mg} / \mathrm{kg})$ of pentobarbital (Hanrim Pharmaceuticals, Seoul, Korea). The common bile duct and a jugular vein were cannulated with polyethylene (i.d. $0.28 \mathrm{~mm}$, o.d. $0.61 \mathrm{~mm}$ ) and silicon tubing (i.d. 0.64 mm, o.d. $1.19 \mathrm{~mm}$ ), respectively. $\left.{ }^{8}\right)$ After APAP was injected to hamsters at a dose of $250 \mathrm{mg} / \mathrm{kg}$, i.p., serial bile and blood samples were collected for up to $360 \mathrm{~min}$. The specific gravity of bile was assumed to be 1.0. Plasma and bile samples were stored in a freezer $\left(-20^{\circ} \mathrm{C}\right)$ until analyzed.

Different groups of hamsters were treated with APAP $(250,350$, or $450 \mathrm{mg} / \mathrm{kg}$, i.p.) for the determination of hepatotoxicity. After APAP treatment the hamsters were fasted for 
$24 \mathrm{~h}$ prior to sacrifice.

Chemicals Taurine, acetaminophen, NADH, D-fructose, L-alanine, L-aspartic acid, $\alpha$-ketoglutaric acid, and pyruvic acid were purchased from Sigma Chemical (St. Louis, MO, U.S.A). Standard metabolites of APAP, such as APAP-glucuronide, APAP-sulfate, APAP-mercapturate, APAP-cysteine, and APAP-GSH, were kindly donated by McNeil Consumer Products (Fort Washington, PA, U.S.A.). All other chemicals and solvents were reagent grade or better.

Assays Concentrations of APAP and its metabolites in plasma and bile were measured using the method of Howie et al. ${ }^{9)}$ Plasma sample was mixed with an aliquot of acetonitrile containing theophylline as an internal standard. After extraction and centrifugation, the resulting supernatant was evaporated to dryness under nitrogen. The residues were diluted with distilled water as necessary before being injected into a Shimadzu HPLC system (Kyoto, Japan) equipped with a UV detector. Parent APAP and its metabolites, i.e., APAP-GSH, APAP-cysteine, APAP-mercapturate, APAP-glucuronide, and APAP-sulfate, were separated in a reverse-phase $\mathrm{C}_{18} \mu$ Bondapack column (Waters Associates, Milford, MA, U.S.A.). APAP and the metabolites were eluted with $1.5 \%$ aqueous acetic acid-methanol $(90: 10)$ at a flow rate of $1.5 \mathrm{ml} / \mathrm{min}$.

The hepatotoxicity of APAP was estimated by measuring the elevation of serum enzyme activities. Alanine aminotransferase (ALT) and aspartate aminotransferase (AST) activities were measured by employing the method of Reitman and Frankel. ${ }^{10)}$ Serum sorbitol dehydrogenase (SDH) activity was determined according to the method of Gerlach. ${ }^{11)}$

Statistical Analysis All results are expressed as mean \pm S.E. Difference between means was determined using Student's $t$-test or one-way ANOVA followed by the NewmanKeuls multiple range test. Acceptable level of significance was established at $p<0.05$ except when otherwise indicated.

\section{RESULTS AND DISCUSSION}

Taurine intake increased the bile flow to approximately $150 \%$ of control (Table 1). Among the numerous physiological functions suggested to be associated with taurine, its role in the secretion and metabolism of bile is relatively well addressed. The increase in bile flow with taurine administration appears to be explained by its effect on bile acid synthesis. Kibe et al. ${ }^{1)}$ observed an induction of cholesterol $7 \alpha$-hydroxylase activity in guinea pigs fed taurine. In the same experiment the half-life of chenodeoxycholic acid (CDC) was decreased by $40 \%$, indicating an increase in the synthesis of bile acids. An increase in the activities of cholesterol $7 \alpha$-hydroxylase and HMG CoA reductase was also demonstrated in hamsters. ${ }^{2)}$ Taurine intake increased the maximum secretory rate of bile acid induced by $\mathrm{CDC}^{12)}$ and alleviated lithocholic acid sulfate-induced cholestasis, ${ }^{13)}$ suggesting that taurine increases the canalicular excretion of bile acids. It has also been suggested that a sulfhydryl compound, such as cysteine and glutathione, modulates the activities of cholesterol $7 \alpha$-hydroxylase and HMG CoA reductase. ${ }^{3,4,14)}$ Therefore, it seems possible that taurine could affect the enzyme activities indirectly by changing the availability of cysteine, a synthetic precursor of taurine. In fact an increase in cysteine levels by taurine was demonstrated in Hep G2 cells. ${ }^{15)}$

In this study there was no difference in bile flow between
Table 1. Effect of Taurine and/or APAP on Bile Flow in Hamsters

\begin{tabular}{|c|c|c|c|c|}
\hline \multirow{2}{*}{$\begin{array}{l}\text { Time } \\
(\min )\end{array}$} & \multicolumn{4}{|c|}{ Bile flow ( $\mu 1 / \mathrm{min} / 100 \mathrm{~g}$ body weight) } \\
\hline & Control & Taurine & APAP & $\begin{array}{l}\text { Taurine } \\
+ \text { APAP }\end{array}$ \\
\hline $0-15$ & $4.38 \pm 0.28 \mathrm{a}$ & $6.08 \pm 0.32 \mathrm{a}$ & $3.94 \pm 0.06 a$ & $8.46 \pm 1.53 b$ \\
\hline $15-30$ & $4.16 \pm 0.12 \mathrm{a}$ & $6.10 \pm 0.47 \mathrm{a}$ & $4.50 \pm 0.32 \mathrm{a}$ & $8.62 \pm 1.16 b$ \\
\hline $30-45$ & $4.15 \pm 0.34 \mathrm{a}$ & $6.49 \pm 0.05 \mathrm{a}, \mathrm{b}$ & $4.34 \pm 0.63 \mathrm{a}$ & $8.46 \pm 1.46 b$ \\
\hline $45-60$ & $4.44 \pm 0.21 \mathrm{a}$ & $6.42 \pm 0.36 b, c$ & $4.30 \pm 0.64 \mathrm{a}, \mathrm{b}$ & $9.17 \pm 1.32 \mathrm{~d}$ \\
\hline $60-75$ & $4.28 \pm 0.27$ & $6.59 \pm 0.53 * * *$ & - & - \\
\hline $75-90$ & $3.94 \pm 0.20$ & $6.52 \pm 0.24 * * *$ & - & - \\
\hline $90-105$ & $3.83 \pm 0.20$ & $5.25 \pm 0.54 * * *$ & - & - \\
\hline $60-120$ & - & - & $3.51 \pm 0.49$ & $7.72 \pm 1.26^{* *}$ \\
\hline $120-180$ & - & - & $3.18 \pm 0.52$ & $8.47 \pm 0.63 * *$ \\
\hline $180-240$ & - & - & $2.93 \pm 0.50$ & $8.47 \pm 0.45 * *$ \\
\hline
\end{tabular}

Hamsters were fed with taurine $(5 \mathrm{~mm})$ in drinking water for 1 week. APAP (250 $\mathrm{mg} / \mathrm{kg}$, i.p.) was injected immediately prior to initiation of bile flow measurement. Value represents mean \pm S.E. for three to six animals. Values followed by different letters are different one from another (one-way ANOVA followed by the Newman-Keuls multiple range test, $p<0.05$ ). Asterisks indicate significant differences from corresponding control (Student's $t$-test, $* *, * * * ; p<0.01,0.001$, respectively).

normal control and APAP-treated animals (Table 1). However, APAP treatment enhanced the elevation of bile flow induced by taurine intake significantly, suggesting that some unknown factor(s) is involved here. The mechanism of APAP-induced increase in bile flow in taurine-fed hamsters remains unclear.

Concentrations of APAP and its major metabolites in bile were not changed by taurine (data not shown). However, the biliary output of APAP and the metabolites was greater in animals fed taurine in accordance with the increase in bile flow (Fig. 1). The most significant increase in biliary output was observed with APAP followed by APAP-GSH (Table 2). The total biliary APAP disposition was more than two-fold greater in taurine-fed hamsters (control, $21 \mathrm{mg} / 360 \mathrm{~min} / \mathrm{kg}$; taurine-fed hamsters, $45 \mathrm{mg} / 360 \mathrm{~min} / \mathrm{kg}$ ).

The plasma APAP concentration was lower (Fig. 2), and the half-life of APAP in plasma was shorter in taurine-fed hamsters, although the difference between the two groups was not statistically significant (control, $75.7 \pm 11.5 \mathrm{~min}$; taurine-fed group, 57.9 $\pm 3.9 \mathrm{~min}$, Student's $t$-test, $p=0.19$ ). However, the area under the curve $(A U C)$ of the plasma APAP concentration was decreased significantly by taurine intake (control, $41.6 \pm 3.6 \mathrm{mg} \cdot \mathrm{min} / \mathrm{ml}$; taurine-fed group, $28.2 \pm 4.5 \mathrm{mg} \cdot \mathrm{min} / \mathrm{ml}$, Student's $t$-test, $p<0.05$ ). This result indicates that taurine intake enhances the elimination of APAP, probably by increasing the biliary disposition of APAP. Plasma concentrations of APAP-glucuronide or APAP-sulfate were not changed, but APAP-GSH and APAPmercapturate were elevated significantly in taurine-fed hamsters.

The total thioether conjugates of APAP reflect the generation of a reactive metabolite, NAPQI, from this drug. ${ }^{7)}$ In hamsters approximately $93 \%$ of APAP-thioether conjugates was shown to be excreted through bile, among which $98 \%$ was APAP-GSH. Therefore, the amount of APAP constituting APAP-GSH in bile may be considered as the amount of toxic APAP-intermediates generated in liver. In this study the biliary APAP-GSH output was doubled, suggesting that the generation of reactive metabolites is enhanced by taurine intake. Metabolic conversion of APAP to the reactive metabolite is catalyzed mostly by CYP2E1, 1A2, and 3A4. Only a 

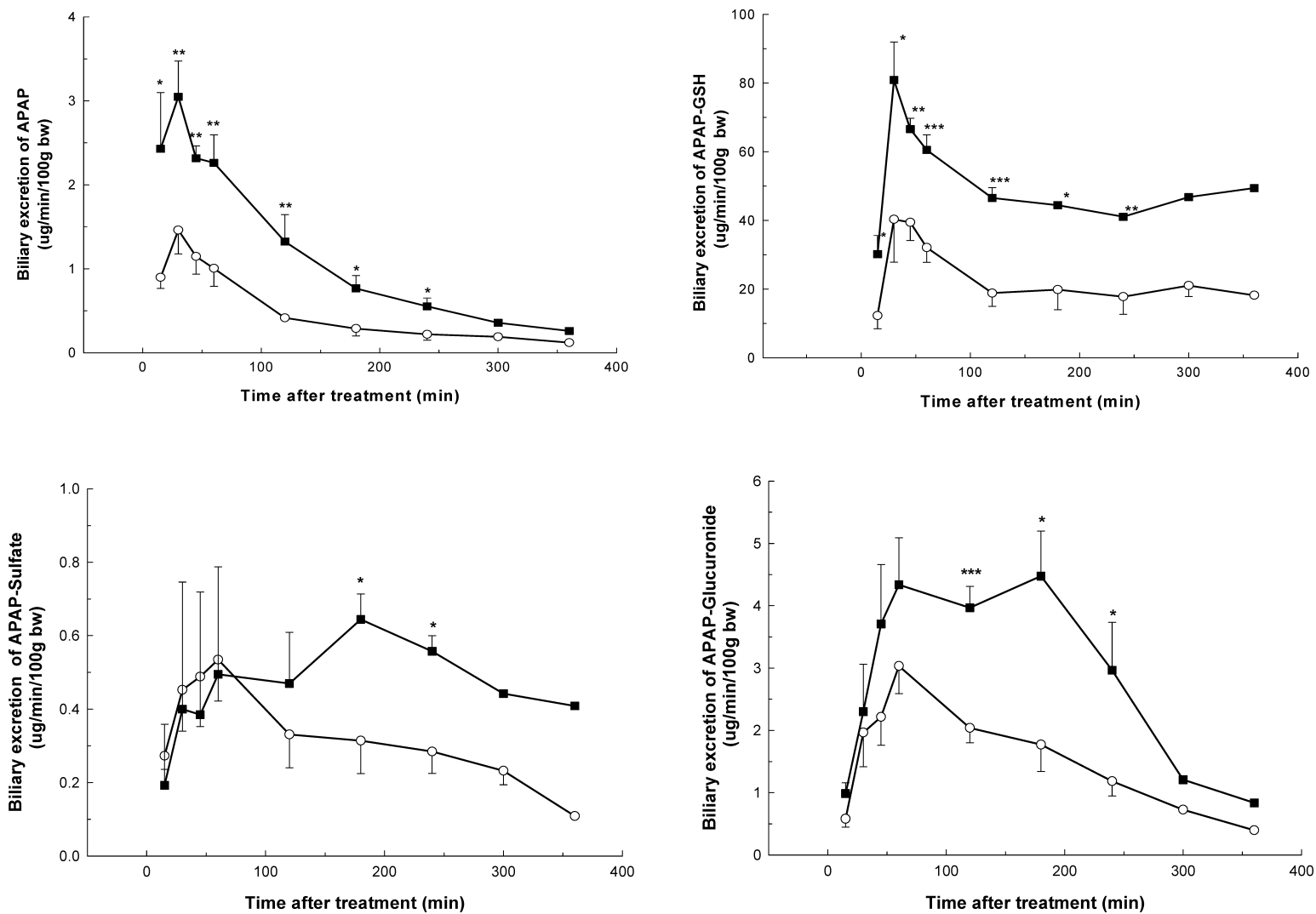

Fig. 1. Biliary Excretion of APAP and Its Metabolites

Value represents mean \pm S.E. for three to six hamsters. Filled squares denote taurine-fed animals; open circles, controls. Asterisks indicate significant differences from control (Student's $t$-test, *,**,***; $p<0.05,0.01,0.001$, respectively).

Table 2. Biliary Disposition of APAP in Hamsters Fed Taurine

\begin{tabular}{lcc}
\hline \hline \multirow{2}{*}{ Metabolite } & \multicolumn{2}{c}{ APAP recovered $(\mu \mathrm{g} / 100 \mathrm{~g}$ body wt $)$} \\
\cline { 2 - 3 } & Control & Taurine \\
\hline APAP & $134 \pm 4$ & $326 \pm 33^{* * *}$ \\
APAP-glucuronide & $197 \pm 18$ & $376 \pm 46^{* *}$ \\
APAP-GSH & $1701 \pm 287$ & $3702 \pm 85^{* * *}$ \\
APAP-sulfate & $57 \pm 10$ & $75 \pm 27$ \\
\hline
\end{tabular}

Hamsters were treated with APAP $(250 \mathrm{mg} / \mathrm{kg}$, i.p.) and bile was collected for 360 min. Value given is $\mu \mathrm{g}$ APAP in each metabolite $/ 100 \mathrm{~g}$ body weight calculated from molecular weights of APAP and the metabolite. Value represents mean \pm S.E. for three to six animals. Asterisks indicate significant differences from control (Student's $t$-test, $* *, * * * ; p<0.01,0.001$, respectively).

few studies have examined the effect of taurine on the activity of CYP subtypes. Zaman et al. ${ }^{16)}$ reported that taurine decreased the metabolism of lidocaine, which is a substrate of CYP3A4, in a perfused rat liver, suggesting a competition of lidocaine and taurine for this CYP subtype. Recently it was shown that taurine enhanced the induction of CYP3A4 by rifampicin in a HepG2 cell line. ${ }^{17)}$ It is suggested that more studies are needed to clarify the effect of this substance on the hepatic drug metabolizing capacity.

An increase in the generation of thioether conjugates indicates that a greater portion of APAP is biotransformed to a reactive metabolite which is responsible for the induction of liver toxicity. Thus, the effect of taurine intake on the hepatotoxicity induced by a dose of APAP was examined. At a dose of 250 or $350 \mathrm{mg} / \mathrm{kg}$, i.p., APAP was shown to be non-toxic to hamsters as measured by the elevation of serum ALT,
Table 3. Effect of Taurine on APAP-Induced Hepatotoxicity

\begin{tabular}{ccccc}
\hline \hline $\begin{array}{c}\text { Enzyme activity } \\
\text { (units/ml) }\end{array}$ & Control & Taurine & APAP & $\begin{array}{c}\text { Taurine } \\
+ \text { APAP }\end{array}$ \\
\hline SDH & $3.8 \pm 1.4$ & $6.1 \pm 2.4$ & $1436 \pm 350$ & $1492 \pm 135$ \\
ALT & $39.6 \pm 2.4$ & $34.6 \pm 0.15$ & $3773 \pm 592$ & $3137 \pm 462$ \\
AST & $38.7 \pm 5.5$ & $34.7 \pm 3.6$ & $2511 \pm 472$ & $1914 \pm 292$ \\
\hline
\end{tabular}

After APAP treatment $(450 \mathrm{mg} / \mathrm{kg}$, i.p. $)$ hamsters were fasted for $24 \mathrm{~h}$ prior to sacrifice. Value represents mean \pm S.E. for four or five animals.

AST, and SDH activities (data not shown). The serum hepatotoxic parameters were increased significantly at an APAP dose of $450 \mathrm{mg} / \mathrm{kg}$, but the taurine-fed group did not demonstrate any changes in the elevation of the serum enzyme activities (Table 3). The reason for the lack of changes in hepatotoxicity despite the greater generation of toxic metabolites is not known. One possibility is that the enhanced biliary excretion of the parent drug reduces the total amount of APAP activated in liver, resulting in a comparable magnitude of hepatotoxicity in both control and taurine-fed hamsters.

In summary, the results of this study showed that taurine intake increases the biliary excretion of APAP and its metabolites significantly. The amount of APAP recovered from the biliary output for $360 \mathrm{~min}$ following the treatment was increased from 8.4 to $18 \%$ of the total APAP dose, greater than $80 \%$ of which was in the form of APAP-GSH. Taurine decreased the AUC of the plasma APAP concentration indicating that elimination of APAP was accelerated. But the elevation of serum ALT, AST, and SDH activities in- 

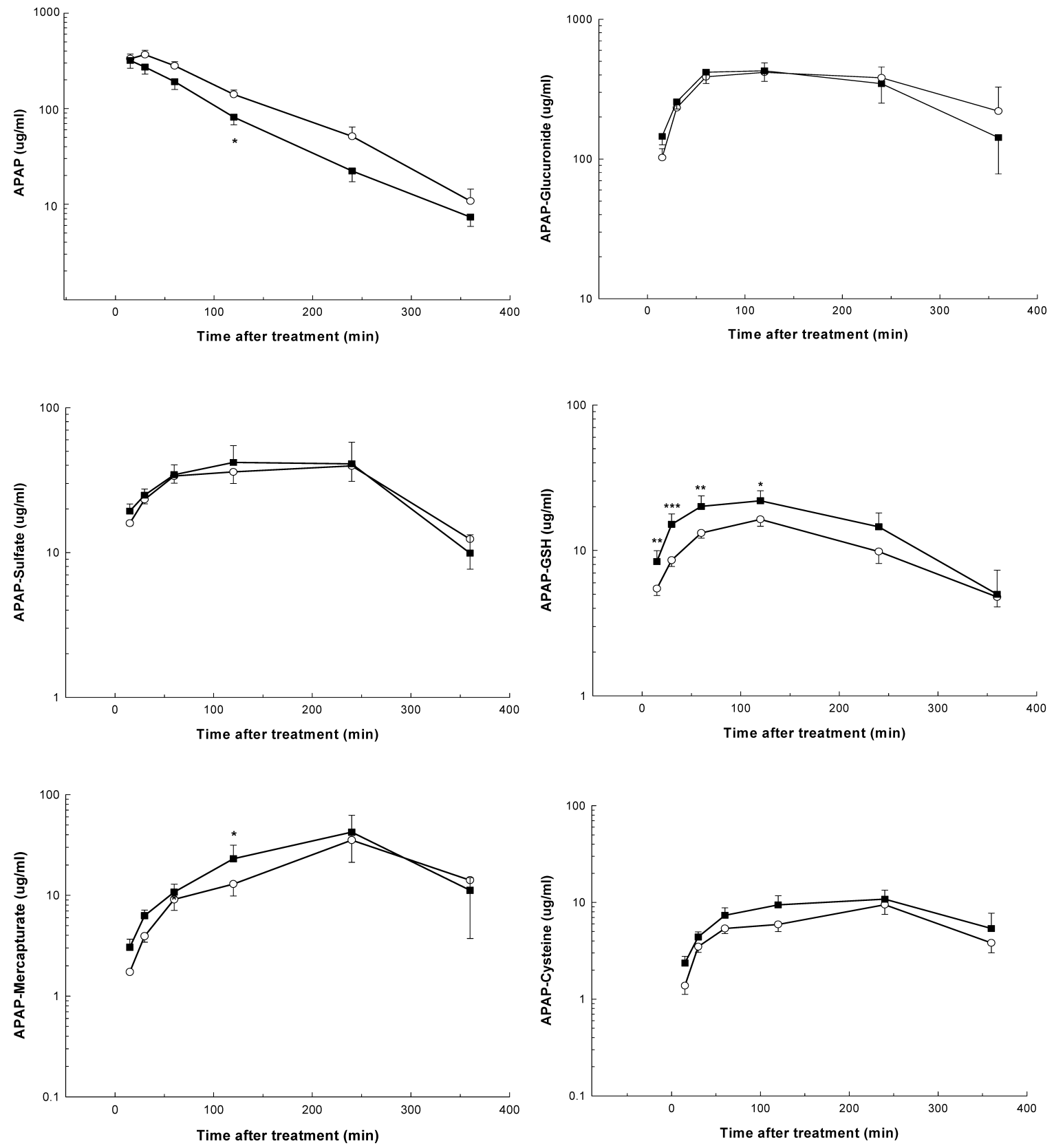

Fig. 2. Plasma Concentrations of APAP and Its Metabolites

Value represents mean \pm S.E. for four hamsters. Filled squares denote taurine-fed animals; open circles, controls. Asterisks indicate significant differences from control (Student's $t$-test, $*, * *, * * * ; p<0.05,0.01,0.001$, respectively).

duced by a toxic dose of APAP was not altered. It is suggested that taurine increases the disposition of APAP by inducing its metabolism through the GSH-dependent pathway and also by enhancing the biliary excretion of this drug and its metabolites.

Acknowledgement This study was supported in part by a grant from the Korea Health 21 R\&D Project, Ministry of Health \& Welfare, Republic of Korea.

\section{REFERENCES}

1) Kibe A., Wake C., Kuramoto T., Hoshita T., Lipids, 15, 224-229 (1980).

2) Bellentani S., Pecorari M., Cordoma P., Marchegiano P., Manenti F., Bosisio E., Fabiani E. D., Galli G., J. Lip. Res., 28, 1021-1027
(1987).

3) Hassan A. S., Hackley J. J., Jeffery E. H., Steroids, 44, 373-380 (1984).

4) Roitelman J., Shechter I., J. Biol. Chem., 259, 870-877 (1984).

5) Patten C. J., Thomas P. E., Guy R. L., Lee M., Gonzales F. J., Guengerich F. P., Yang C. S., Chem. Res. Toxicol., 6, 511-518 (1993).

6) Kim Y. C., Lee S. J., Toxicology, 128, 53-61 (1998).

7) Madhu C., Gregus Z., Klaassen C. D., J. Pharmacol. Exp. Ther., 248, 1069-1077 (1989).

8) Cocchetto D. M., Bjornsson T. D., J. Pharm. Sci., 72, 465-492 (1983).

9) Howie D., Andriaenssens P. I., Prescott L. F., J. Pharm. Pharmacol., 29, 235-237 (1977).

10) Reitman S., Frankel S. K., Am. J. Clin. Pathol., 28, 56-63 (1957).

11) Gerlach U. E., "Methods in Enzymatic Analysis," Vol. 3, ed. by Bergmeyer H. U., Verlag Chemie, Weinheim, 1983, pp. 112-117.

12) Belli D. C., Fournier L., Lepage G., Yousef I. M., Roy C. C., Pediatr. Res., 24, 34-37 (1988). 
13) Belli D. C., Roy C. C., Fournier L., Tuchweber B., Giguere R., Yousef I. M., Liver, 11, 162-169 (1991).

14) Danielsson H., Kalles I., Wikvall K., J. Biol. Chem., 259, 4258-4262 (1984).

15) Stephan Z. F., Lindsey S., Hayes K. C., J. Biol. Chem., 262, 60696073 (1987).
16) Zaman N., Tam Y. K., Jewell L. D., Coutts R. T., Drug Metab. Dis., 24, 534-541 (1996).

17) Takahashi K., Matsuda H., Kinoshita K., Matsunaga N., Sumita A., Matsuda T., Takahashi K., Azuma J., Adv. Exp. Med. Biol., 526, 237244 (2002). 